\title{
Coronary-Cavitary Fistula of the Circumflex Coronary Artery to the Left Atrium as a Probable Etiology of Heart Failure: A Case Report
}

\author{
Fístula Coronário-Cavitária da Artéria Coronária Circunflexa para o Átrio Esquerdo como Provável Etiologia de \\ Insuficiência Cardíaca: Relato de Caso
}

Roberțo Carlos Alvarez Coello, ${ }_{1}$, Vițor Coutinho Andrade ${ }^{1}$, Santiago Andrés Castro Vintimilla ${ }^{1}$, Vagner Madrini
Júnior, José Felix Alvarez Ramirez
Instituto do Coração, School of Medicine, University of São Paulo, São Paulo, Brazil

\begin{abstract}
Coronary artery fistulas have a low incidence and are often diagnosed by echocardiography or coronary computed tomography angiography, although coronary angiography is the gold standard. They commonly originate in the right coronary artery, with drainage to low-pressure chambers being the most frequent finding. Treatment can be expectant, surgical, or percutaneous. This report describes a case of a rare presentation of heart failure due to a fistula of the circumflex coronary artery with drainage into the left atrium.
\end{abstract}

\section{Introduction}

Coronary fistulas (CF) are direct connections from one or more coronary arteries to cardiac chambers or great vessels. ${ }^{1}$ They originate from the right coronary artery in up to $50 \%$ of cases and from the left anterior descending artery in $42 \%$ of cases. Drainage usually occurs into low-pressure chambers, most commonly the right ventricle $(41 \%)$, right atrium $(26 \%)$, or pulmonary artery $(15 \%-17 \%) .^{2}$ Congenital anomalies are more prevalent than acquired anomalies, being found on up to $0.2 \%$ of routine coronary angiographies. ${ }^{1}$

Most patients are asymptomatic, but they can present with fatigue, dyspnea on exertion, palpitations, and angina. Continuous heart murmurs can be heard. ${ }^{1,2}$

The complementary electrocardiography findings are usually normal, although they may present characteristics of left atrial overload and ischemic changes. Diagnosis is usually made by echocardiography, especially when the involved coronary artery is dilated or in the presence of high-output coronary flow. Coronary computed tomography angiography and cardiac magnetic resonance have been increasingly used for anatomical assessments, but coronary angiography is the gold standard for diagnosis, showing important anatomical details for treatment. ${ }^{3}$

Management remains controversial; however, surgical or percutaneous closure is recommended for symptomatic patients or cases of significant shunts. ${ }^{2,4}$

\section{Keywords}

Fistula, Heart atria, Heart failure.

Mailing Address: Roberto Carlos Alvarez Coello •

E-mail: rcacoello8@gmail.com / rcacoello@hotmail.com

Manuscript received 5/26/2021; revised 7/23/2021; accepted 9/16/2021

DOI: $10.47593 / 2675-312 X / 20213404$ eabc220

\section{Case report}

A previously hypertensive 79-year-old man with permanent atrial fibrillation and heart failure with a reduced ejection fraction but no defined etiology was admitted with unstable bradycardia for permanent pacemaker implantation. Echocardiography performed to investigate the heart failure showed a 78-mm left atrium, 10-mm septum, $10-\mathrm{mm}$ posterior wall, $65-\mathrm{mm}$ end diastolic diameter, $40 \%$ left ventricular ejection fraction, mildly hypokinetic right ventricle, moderate secondary mitral regurgitation, severe tricuspid regurgitation, and $60 \mathrm{mmHg}$ pulmonary artery systolic pressure. A thrombus was visualized in the left atrial appendage. No fistulous tract was evidenced, probably because a fistula was not suspected at that time.

Coronary angiography revealed non-obstructive lesions with no indications for intervention. The injection of contrast into the left coronary artery suggested a mass in the region corresponding to the left atrium (no contrast uptake inside), with extravasation of intracavitary contrast medium ("cigarette smoke" sign) from the circumflex artery (Figures 1 and 2).

Complementary coronary computed tomography angiography showed a thrombus in the left atrial appendage in addition to mild left atrial hyperattenuating signs adjacent to the proximal/middle portion of the circumflex artery (Figure 3).

\section{Discussion}

CF was first described in 1886, and the first successful surgical correction was performed in $1947 .{ }^{4}$ Its incidence among congenital heart diseases is low, totaling only $0.2 \%-$ $0.4 \%$, with the congenital origin being more common than the acquired one. ${ }^{1}$

Epidemiologically, right CFs are the most frequent ( $50 \%-70 \%$ of cases), but they may also occur in the left coronary artery or both arteries. ${ }^{1}$ Drainage usually occurs into low-pressure chambers. Thus, communication with the right chambers has a higher incidence, $39 \%-41 \%$ for the right ventricle, $26 \%-33 \%$ for the right atrium, and $15 \%-20 \%$ for the pulmonary artery. In contrast, fistulas to the left ventricle are very rare, with an incidence of only $3 \% .{ }^{1,2}$

A CF can lead to coronary artery dilation due to increased flow and pulmonary arterial hypertension, blood shifting from the left to the right, congestive heart failure, and myocardial ischemia due to coronary steal phenomenon, thrombosis, or fistula aneurysm. ${ }^{2}$

The patient's clinical condition depends on the magnitude 


\section{Case Report}

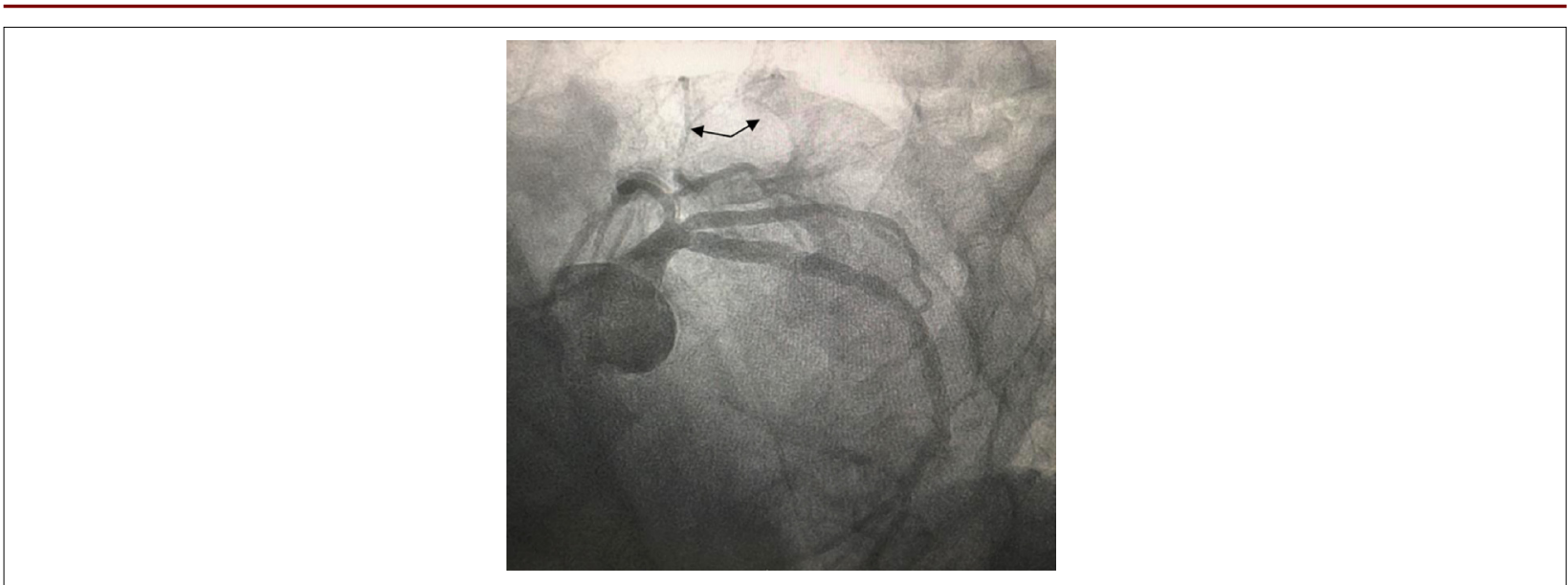

Figure 1 - Coronary angiogram showing left anterior oblique caudal projection (spider). The arrows indicate the ascending branches in a halo communicating with the left atrium.

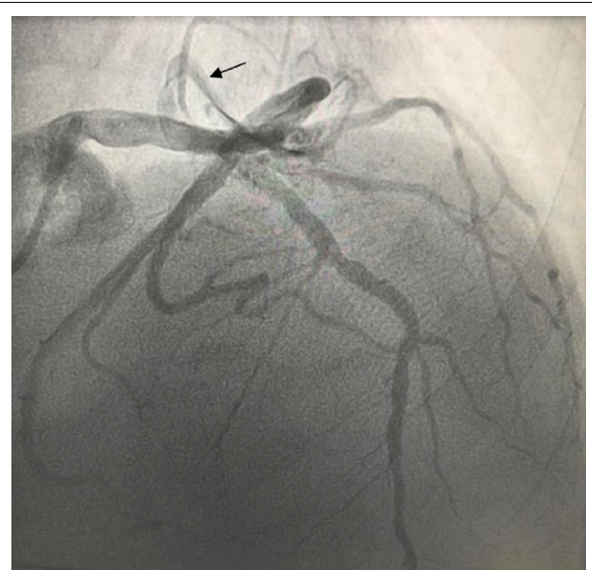

Figure 2 - Coronary angiogram showing a right anterior oblique cranial projection. The arrow indicates the communication of the circumflex artery with the left atrium ("cigarette smoke" sign in a halo around the thrombus).

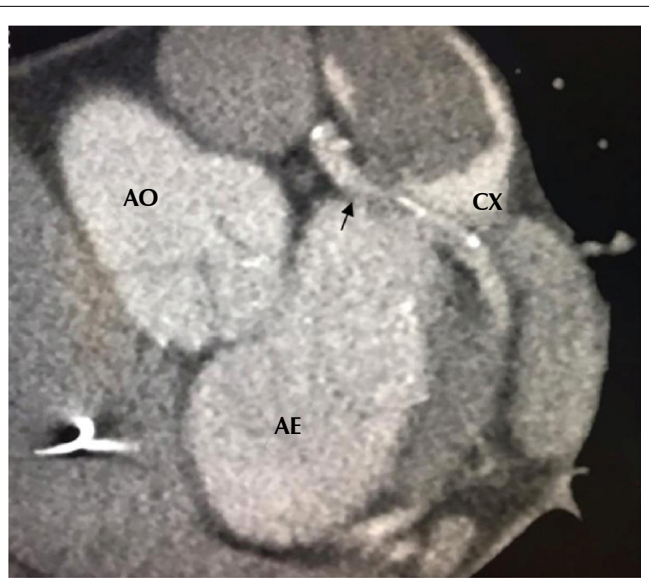

$A E$, left atrium; $A O$, aorta; $C X$, circumflex artery .

Figure 3 - Coronary computed tomography angiogram. The arrow indicates the presence of a fistula communicating between the proximal/middle portion of the circumflex coronary artery and the left atrium. 
of blood flow through the fistula as well as its location. Most carriers are asymptomatic, but the most common clinical presentation is heart failure or dyspnea on exertion. Continuous, superficial, and intense heart murmur in the classic location of a persistent ductus arteriosus may be a finding on physical examination. It can also present as clinical signs of angina due to a decreased coronary flow reserve caused by "leakage flow."1,2,5

The differential diagnosis of coronary arteriovenous fistula includes conditions that dilate the coronary arteries, the presence of collateral vessels, and a continuous heart murmur, such as a patent ductus arteriosus, ruptured sinus of Valsalva aneurysm, or pulmonary fistula. ${ }^{2}$

Fistulas can be diagnosed by echocardiography with the visualization of a dilated coronary artery or by Doppler evaluation of the fistula itself. Imaging tests such as coronary computed tomography angiography and cardiac magnetic resonance imaging can play a significant role in the diagnosis and definition of the therapeutic strategy for this pathology. However, coronary angiography is the method of choice for diagnosis, although some details of the relationship between the fistula and other structures may not be visualized with this technique. ${ }^{3}$

Spontaneous fistula closure is very rare, although cases have been described. Small asymptomatic fistulas require no treatment and are followed up for complications. The guidelines of the American College of Cardiology and the American Heart Association recommend closing fistulas larger than $250 \mathrm{~mm}$ regardless of symptoms and addressing all

\section{Referências}

1. Mendonça RM, Bandeira RL, Fonseca FJ, Macedo Filho R. Fístula coronariana para ventrículo esquerdo: diagnóstico por tomografia computadorizada. Arq Bras Cardiol. 2011;97(4):e82-e85

2. Nepal S, Annamaraju P. Coronary arteriovenous fistula. 2021 Aug 11. In: StatPearls [Internet]. Treasure Island (FL): StatPearls Publishing; 2021 Jan-. PMID: 32119505.

3. Chen ML, Lo HS, Su HY, Chao IM. Coronary artery fistula: assessment with multidetector computed tomography and stress myocardial single photon symptomatic fistulas, including cases of myocardial ischemia, arrhythmia, ventricular dysfunction, and endarteritis. The fistula can be closed using a percutaneous transcatheter approach or surgery. ${ }^{5}$

In the present case, the main etiological hypothesis of heart failure was defined as the coronary-cavitary fistula, probably of congenital origin, due to the intracavitary flow that generated continuous blood volume overload in the left chambers and subsequent remodeling of the right cavities with consequent biventricular dysfunction. Surgery was suggested but then discarded due to the high surgical risk and the patient's fragile status. Percutaneous intervention was also discarded due to the high risk of left atrial thrombus embolization at that time; thus, oral anticoagulants and subsequent clinical follow-up was chosen.

\section{Authors' contributions}

Research conception and design: Coello RCA; data collection: Coello RCA, Andrade VC, and Vintimilla SAC; data analysis and interpretation: Madrini Junior $V$; manuscript writing: Coello RCA, Andrade VC, and Vintimilla SAC; critical review of the manuscript for important intellectual content: Madrini Júnior $\mathrm{V}$ and Ramirez JFA.

\section{Conflict of interest}

The authors have declared that they have no conflict of interest.

emission computed tomography. Clin Nucl Med. 2009;34(2):96-8. doi: 10.1097/RLU.0b013e318192c497.

4. Parra-Bravo JR, Beirana-Palencia LG. Fístula de arteria coronaria derecha drenando al ventrículo derecho. Hallazgos ecocardiográficos y manejo intervencionista. Arch Cardiol Mex. 2003;73(3):205-11. Spanish. PMID: 14635481.

5. Challoumas D, Pericleous A, Dimitrakaki IA, Danelatos C, Dimitrakakis G. Coronary arteriovenous fistulae: a review. Int J Angiol. 2014;23:1-10. doi: 10.1055/s-0033-1349162. 